\title{
Directed and Elliptic Flow at RHIC
}

\author{
A.H. Tang $\dagger$ for the STAR Collaboration $\ddagger$ \\ $\dagger$ NIKHEF and BNL \\ Physics Department, P.O. Box 5000, Brookhaven National Laboratory, Upton, \\ NY 11973, aihong@bnl.gov
}

\begin{abstract}
We present the directed flow measurement $\left(v_{1}\right)$ from $\mathrm{Au}+\mathrm{Au}$ collisions at $\sqrt{s_{\mathrm{NN}}}=62 \mathrm{GeV}$. Over the pseudorapidity range we have studied, which covers $\eta$ from -1.2 to 1.2 and $2.4<|\eta|<4$, the magnitude of $v_{1}$ for charged particles is found to increase monotonously with pseudorapidity for all centralities. No " $v_{1}$ wiggle", as predicted by various theoretical models, is observed at midrapidity. Elliptic flow $\left(v_{2}\right)$ from moderate high $p_{t}$ particles $(3-6 \mathrm{GeV} / c)$ at $\sqrt{s_{\mathrm{NN}}}=200 \mathrm{GeV}$ is presented as a function of impact parameter. It is found that models that are based on jet quenching alone appear to underpredict $v_{2}$ at moderate high $p_{t}$, while the model that incorporates both, recombination and fragmentation, describes the data better.
\end{abstract}

\section{Introduction}

In non-central heavy ion collisions, the azimuthal distribution of emitted particles with respect to the reaction plane is not uniform. It can be characterized [1] by Fourier coefficients

$$
v_{n}=\langle\cos n(\phi-\psi)\rangle
$$

where $\phi$ denotes the azimuthal angle of an emitted particle, $\psi$ is the orientation of the reaction plane, and $n$ denotes the harmonic.

The first Fourier coefficient, $v_{1}$, referred to as directed flow, describes the sideward motion of the fragments in ultra-relativistic nuclear collisions and it carries early information from the collision. Its shape at midrapidity is of special interest because it might reveal a signature of a possible phase transition from normal nuclear matter to a quark-gluon plasma 2]. Because of its importance, directed flow recently has attracted increased attention of both experimentalists and theoreticians [3, 4, 5, 6, 7, 2, 8, 9, In the paper [4] that reports the first $v_{1}$ measurement at RHIC, the shape of $v_{1}$ at midrapidity is left ambiguous, due to the large statistical error. It is now possible to answer this question with the large statistics obtained during RHIC run of 2004.

Elliptic flow $\left(v_{2}\right)$ is caused by the initial geometric deformation of the reaction region in the transverse plane. At low transverse momentum, roughly speaking, large values of $v_{2}$ are considered signatures of hydrodynamic behavior. At large transverse $\ddagger$ For the full author list and acknowledgements see Appendix "Collaborations" in this volume. 
momentum, in a jet quenching picture [10, elliptic flow results from that jets emitted out-of-plane suffer more energy loss than those emitted in-plane. In an extreme case of jet quenching, particles are all emitted from the surface, as a consequence of that, $v_{2}$ is dominated by the geometry of the source. Thus it is interesting to study $v_{2}$ at large $p_{t}$, where the hydrodynamic description of the system is expected to break down and jet quenching is expected to happen, as a function of impact parameter. Such study should give us a good constraint on various models.

\section{Directed flow}

The data for the $v_{1}$ analysis is based on the fourth year of operation of the Relativistic Heavy Ion collider (RHIC) at $\sqrt{s_{\mathrm{NN}}}=62.4 \mathrm{GeV}$. The STAR detector [1] main Time Projection Chamber (TPC [12]) and two forward TPCs (FTPC [13]) were used in the analysis. The data set consists of about 5 million minimum bias $\mathrm{Au}+\mathrm{Au}$ events. The analysis is done with two methods, namely, three-particle cumulant method [14] and event plane method with mixed harmonics [7].

Fig. 11 shows $v_{1}$ from the three-particle cumulants method and the event plane method with mixed harmonics as a function of pseudorapidity $(\eta)$. Both methods are based on three particle correlations and they should give the same result, which is confirmed by the plot. The plot shows that over the pseudorapidity range we have studied, which covers $\eta$ from -1.2 to 1.2 and $2.4<|\eta|<4$, the magnitude of $v_{1}$ for charged particles is found increasing monotonically with pseudorapidity for all centralities. No " $v_{1}$ wiggle" is observed at midrapidity as predicted by various theoretical models [2]. The centrality dependence of $v_{1}$ is shown in Fig. 2] As expected, in all pseudorapidity regions, $v_{1}$ decreases with centrality. It is noticed that $v_{1}$ in the forward region decreases faster with centrality than that at midrapidity.

Limiting fragmentation [15] has successfully explained the spectra and some flow results in the forward region [16, 4]. In Fig. 3] we show $v_{1}$ results from three different energies in the projectile frame relative to their respective beam rapidities. They look similar in the forward region.

\section{Elliptic flow}

The large value of the elliptic flow at high $p_{t}$ [17] and the strong suppression of back-toback high $p_{t}$ jet-like correlations [18] support the jet-quenching scenario qualitatively, however, the amount of elliptic flow observed at high $p_{t}$ for collisions at $\sqrt{s_{\mathrm{NN}}}=130 \mathrm{GeV}$ seems to exceed the values expected in the case of complete quenching [19]. Extreme quenching leads to emission of high- $p_{t}$ particles predominantly from the surface, and in this case $v_{2}$ would be fully determined by the geometry of the collision. This hypothesis can be tested by studying the centrality dependence of $v_{2}$ for high- $p_{t}$ particles.

Figure 4 shows $v_{2}$ in the $p_{t}$-range of $3-6 \mathrm{GeV} / c$ (where $v_{2}$ is approximately maximal and constant) versus impact parameter from $\mathrm{Au}+\mathrm{Au}$ collision at $200 \mathrm{GeV}$. For more 


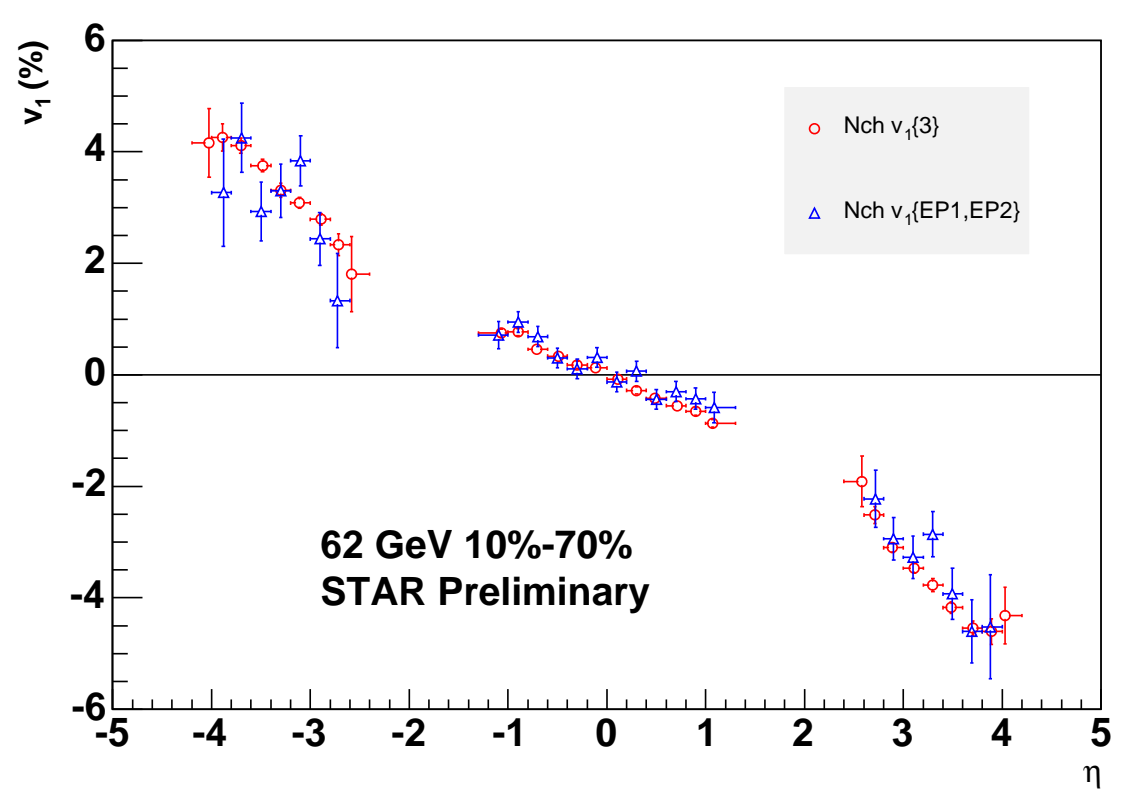

Figure 1. $v_{1}$ from three particle cumulant method (circles) and event plane method with mixed harmonics (triangles) as a function of pseudorapidity. Errors are statistical. The event plane result is from Markus Oldenburg.

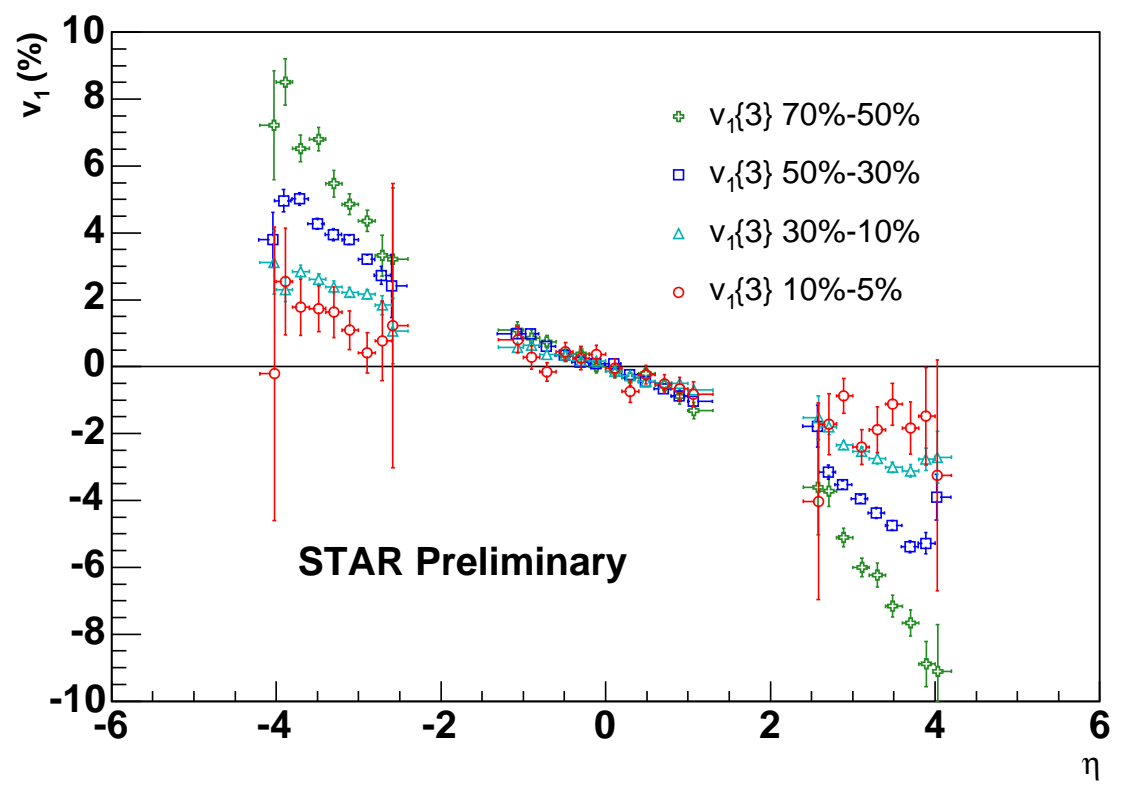

Figure 2. $v_{1}$ from three particle cumulant method as a function of pseudorapidity for four centrality bins. Errors are statistical. 


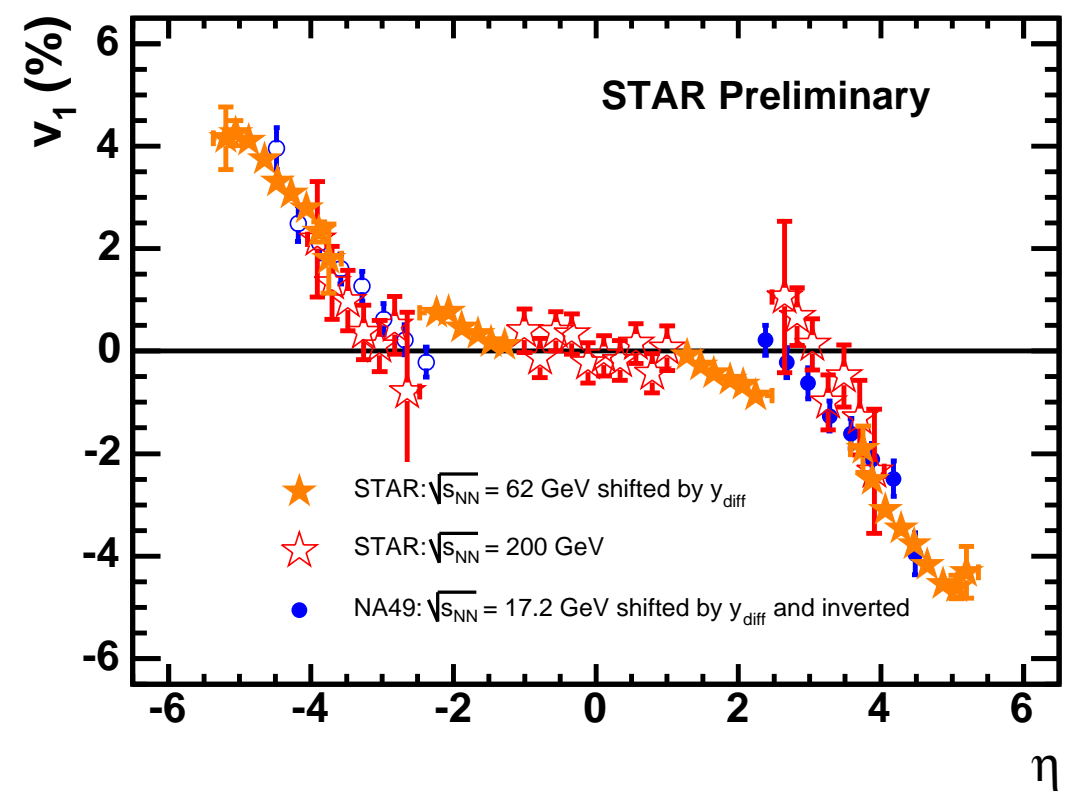

Figure 3. The values of $v_{1}$ from charged particles for $\mathrm{Au}+\mathrm{Au}$ collisions at $200 \mathrm{GeV}$ (open stars) and $62 \mathrm{GeV}$ (solid stars) plotted as a function of pseudorapidity. Also shown are the results from NA49 (circles) for pions from $158 \mathrm{~A} \mathrm{GeV} \mathrm{Pb}+\mathrm{Pb}$ midcentral (12.5\%-33.5\%) collisions plotted as a function of rapidity. The open circles of NA49 have been reflected about midrapidity. The NA49 and $62 \mathrm{GeV}$ points have been shifted plus or minus by the difference to $200 \mathrm{GeV}$ in the beam rapidities. All results are from analyses involving three-particle cumulants, $v_{1}\{3\}$.

description of the data set see [20. The values of the impact parameters were obtained using a Monte Carlo Glauber calculation [21. The measured values of $v_{2}\{4\}$ are compared to various simple models of jet quenching. The upper curve corresponds to a complete quenching scenario, in which particles are emitted from a hard shell [22, 19]; this yields the maximum values of $v_{2}$ which are possible under a surface emission assumption. A more realistic calculation corresponds to a parameterization of jet energy loss in a static medium where the absorption coefficient is set to match the suppression of the inclusive hadron yields [23. The density distributions of the static medium are modeled using a step function (following [24) and a more realistic WoodsSaxon distribution (following [25]). The corresponding $v_{2}$ values are shown as the upper and lower band, respectively. The lower and upper boundaries of bands correspond to an absorption that gives a suppression factor of 3 and 5 [23, respectively, in central collisions. Over the whole centrality range, the measured $v_{2}$ values are much larger compared to calculations. Taking into account that this measurement is dominated by the yield at lower $p_{t}(3 \mathrm{GeV} / \mathrm{c})$, the recombination of quarks might be responsible for the difference. Indeed a model that combines the mechanism of both recombination and fragmentation [26] gives a $v_{2}$ result that is larger than other models and is close to the 


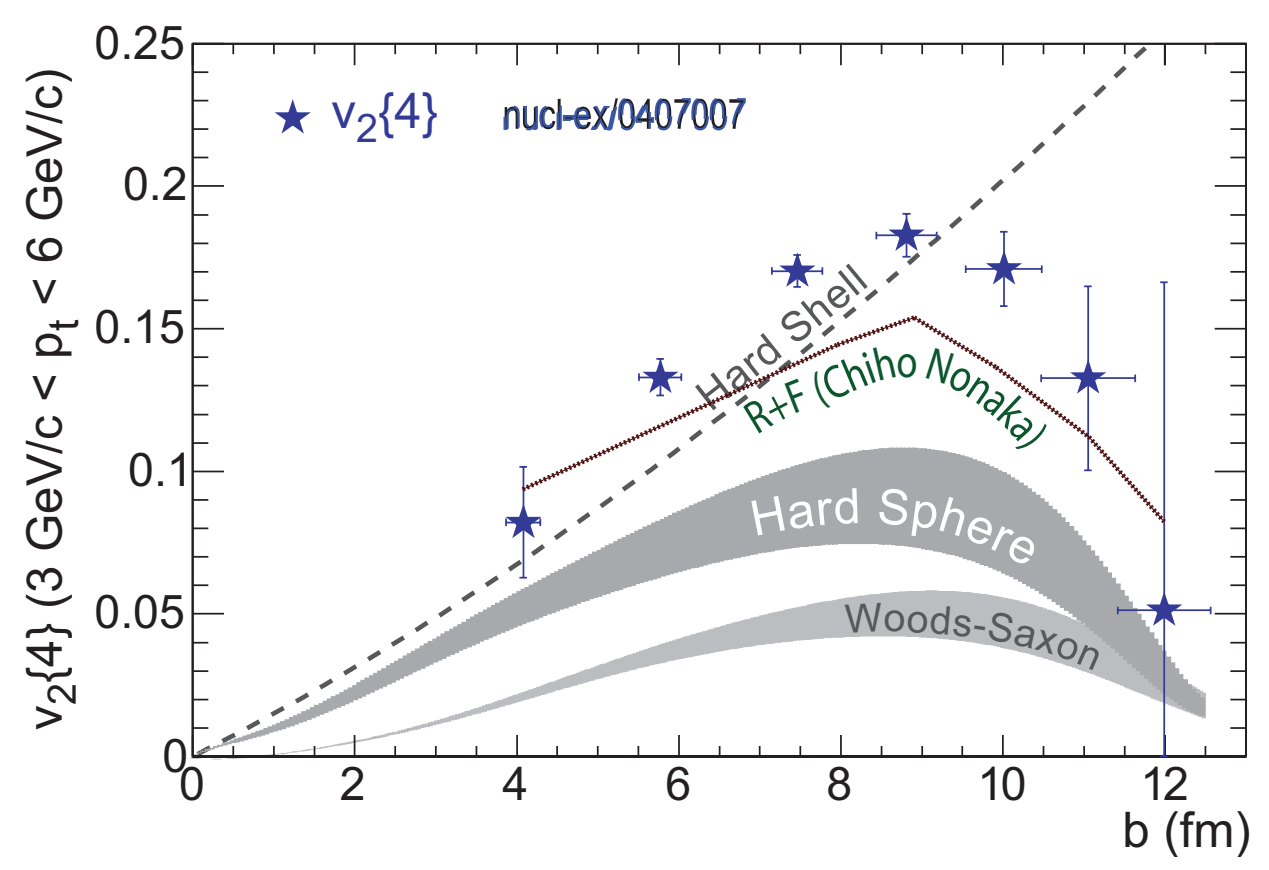

Figure 4. $\quad v_{2}$ at $3 \leq p_{t} \leq 6 \mathrm{GeV} / c$ versus impact parameter, $b$, compared to jet quenching models and the model that incorporate both recombination and fragmentation. The data is from $200 \mathrm{GeV} \mathrm{Au+Au}$ collisions. See ref. [26] for $\mathrm{R}+\mathrm{F}$ calculation.

data (see $\mathrm{R}+\mathrm{F}$ curve in Figure 4). It would be useful to have a quantitative estimate of the systematical uncertainty in this calculation so that the remaining discrepancy can be understood.

\section{Summary}

In summary, we have presented the $v_{1}$ measurement from $\mathrm{Au}+\mathrm{Au}$ collisions of $62 \mathrm{GeV}$ at RHIC. Over the pseudorapidity range we have studied, which covers $\eta$ from -1.2 to 1.2 and $2.4<|\eta|<4$, the magnitude of $v_{1}$ for charged particles is found to increase monotonically with pseudorapidity for all centralities. No " $v_{1}$ wiggle" for charged particles, as predicted by various theoretical models, is observed at midrapidity. Viewed in the projectile frame, $v_{1}$ from three different energies $(17.2 \mathrm{GeV}, 62.4 \mathrm{GeV}$ and 200 $\mathrm{GeV}$ ) looks similar, in support of limiting fragmentation hypothesis. We have studied $v_{2}$ for moderate high $p_{t}$ particles from $\mathrm{Au}+\mathrm{Au}$ collisions at $200 \mathrm{GeV}$, as a function of centrality, and found that models that are based on jet quenching alone underpredict $v_{2}$. A model that combines both recombination and fragmentation describe the data better.

\section{References}

[1] Poskanzer A.M. and Voloshin 1998 Phys. Rev. c 581671 
[2] Brachmann J et al 2000 Phys. Rev. C 61024909

Bleicher M and Stöcker H 2002 Phys. Lett. B 526309

Csernai L P and Roehrich D 1999 Phys. Lett. B 458454

Snellings R J M, Sorge H, Voloshin S A, Wang F Q and Xu N 2000 Phys. Rev. Lett. 842803

[3] Alt C et al [NA49 Collaboration] 2003 Phys. Rev. C 68034903

[4] Adams J. et al [STAR Collaboration] 2004 Phys. Rev. Lett. 92062301

[5] Tonjes M.B. et al [PHOBOS Collaboration] 2004 J. Phys. G 30 S1243-S1246

[6] Tang A.H. et al [STAR Collaboration] 2004 J. Phys. G 30 S1235-S1238

[7] Oldenburg M.D. et al [STAR Collaboration] nucl-ex/0403007

[8] Stoecker H. Proceedings for RBRC Workshop "New Discoveries at RHIC" nucl-th/0406018

[9] Chen L., Creco V., Ko C.M. and Kolb P.F. nucl-th/0408021

[10] Snellings R.J., Poskanzer A.M. and Voloshin S.A nucl-ex/9904003 Wang X.N. 2001 Phys. Rev. C 63054902

[11] Ackermann K.H. et al 2003 Nucl. Instrum. Method A 499624

[12] Anderson M. et al 2003 Nucl. Instrum. Method A 499659

[13] Ackermann K.H. et al 2003 Nucl. Instrum. Method A 499713

[14] Borghini N, Dinh P M and Ollitrault J.-Y. 2002 Phys. Rev. C 66014905

[15] J.Benecke, T.T. Chou, C-N. Yang and E. Yen, Phys. Rev. 188, 2159 (1969)

[16] Back B.B. et al [PHOBOS Collaboration] 2003 Phys. Rev. Lett. 91052303 Back B.B. et al [PHOBOS Collaboration] nucl-ex/0406012

[17] Adler C et al STAR collaboration 2003 it Phys. Rev. Lett. 90, 032301

[18] Adler C et al STAR collaboration 2003 Phys. Rev. Lett. 90082302

[19] Shuryak E.V. 2002 Phys. Rev. C 66027902

[20] Adams J. et al [STAR Collaboration] nucl-ex/0407007

[21] Back B.B. et al 2002 Phys. Rev. C 65 031901(R)

Adcox k. et al 2001 Phys. Rev. Lett. 863500

Bearden I.G. et al 2001 Phys. Lett. B $\mathbf{5 2 3} 227$

[22] Voloshin S. A. 2003 Nucl. Phys. A 715 379c

[23] Adams J. et al [STAR Collaboration] 2003 Phys. Rev. Lett. 91172302

[24] Xin-Nian Wang, private communication. Calculation is based on the framework of nucl-th/0305010

[25] Drees A., Feng H. and Jia J. nucl-th/0310044

[26] C. Nonaka, Talk given at Hot Quark 2004 Conference, See this proceeding 